Bull. Chem. Soc. Ethiop. 2013, 27(3), 377-386.

Printed in Ethiopia

ISSN 1011-3924

DOI: http://dx.doi.org/10.4314/bcse.v27i3.6

(c) 2013 Chemical Society of Ethiopia

\title{
SYNTHESES AND PYROLYTIC STUDIES OF SALICYLATE DERIVATIVES OF HETEROTRINUCLEIC [AI $\left.{ }^{\mathrm{III}}-\mathrm{Sn}^{\mathrm{II}}-\mathrm{B}^{\mathrm{III}}\right]-\mu-O X O I S O P R O P O X I D E$
}

\author{
Rajesh Kumar ${ }^{*}$ \\ Department of Chemistry, HCTM Technical Campus, Kaithal-136027 Haryana, India
}

(Received July 26, 2012; revised May 16, 2013)

\begin{abstract}
New salicylate derivatives of heteronucleic- $\mu$-oxoisopropoxide $\left[\mathrm{SnO}_{2} \mathrm{AlB}\left(\mathrm{OPr}^{\mathrm{i}}\right)_{4}\right]$ have been synthesized by the thermal condensation of $\mu$-oxoisopropoxide and methyl/ethyl/phenyl/phenyl ethyl salicylates in different molar ratios (1:1-1:2) yielding the compounds of the type $\left[\mathrm{SnO}_{2} \mathrm{AlB}\left(\mathrm{OPr}^{\mathrm{i}}\right)_{4-\mathrm{n}}(\mathrm{RSAL})_{\mathrm{n}}\right]$ (where $\mathrm{n}$ is $1-2$ and RSAL = salicylate anion), respectively. The complexes have been characterized by elemental, spectral (IR, ${ }^{1} \mathrm{H},{ }^{13} \mathrm{C},{ }^{27} \mathrm{Al},{ }^{119} \mathrm{Sn}$ and $\left.{ }^{11} \mathrm{~B} \mathrm{NMR}\right)$, thermal and molecular weight measurement. The complexes are hydrolysed by hydrothermally assisted sol-gel technique and gave hydrolyzed product. Thermal studies of these products are made which favor the formation of multicomponent oxides. The studies reveal that salicylate derivatives are polymeric in nature and low susceptible to hydrolysis as compared to parent compound and may prove excellent precursors for the mixed metal oxides.
\end{abstract}

KEY WORDS: Heteronucleic- $\mu$-oxoalkoxides, Aluminium, Tin, Boron, Salicylates, TG analysis

\section{INTRODUCTION}

The investigation and the use of heterometallic alkoxides as single-source molecule precursors for synthesis of oxides have seen a rapid growth during the last more than one and half decade. The control of particle size and the morphology of the oxide are of crucial importance nowadays both from the fundamental and industrial point of view [1]. Heterometallic- $\mu$-oxoalkoxides are often associated with more accessible precursors such as carboxylates, Salicylates and $\beta$ diketonates in chemical routes to intricate mixed metal oxides. The mixed metal oxides of the type $\mathrm{MAl}_{2} \mathrm{O}_{4}(\mathrm{M}=\mathrm{Ca}, \mathrm{Mg}, \mathrm{Mn}, \mathrm{Co}, \mathrm{Fe}, \mathrm{Ni}, \mathrm{Zn})$ obtained from heterometallic- $\mu$-oxoalkoxides precursors [2-5] have found use to new ceramics $[6,7]$ and of medicinal importance such as for absorbing harmful chemicals [8] and gases such as $\mathrm{SO}_{2}, \mathrm{CCl}_{4}$ and decontaminating chemical warfare agents [9]. Interestingly $\mathrm{MgAl}_{2} \mathrm{O}_{4}$ prepared from $\left[\mathrm{MgO}_{2} \mathrm{Al}_{2}\left(\mathrm{OPr}^{\mathrm{i}}\right)_{4}\right]_{2}$ have been used to destructively adsorb paraxon [diethyl-4-nitrophenol phosphate (DNPP)] [10]. Nanocrystalline metal oxides of magnesium, calcium, aluminium, and transition metals have proven to be exceptional adsorbents and catalysts due to their smaller size and high surface area [11-15]. Core/shell bimetallic mixtures, such as $\mathrm{MgO}$ coated with $\mathrm{V}_{2} \mathrm{O}_{3}$, possess their own unique sorption and catalytic properties [16]. In view of the solubility of metal alkoxides [17, 18] and oxoalkoxides $[19,20]$ in organic solvents, these materials are strongly preferred as precursors in sol-gel processes. In heterometallic- $\mu$-oxoalkoxides M-O-M' linkage is present, which makes the M-O-M' bond strong and stable as compared to other precursors such as hetero binuclear and trinuclear alkoxides where metal-metal are linked through -OR groups which are highly susceptible to hydrolysis (due to the electrophilic nature of metal) and undergo cleavage of MOR bond to form metal hydroxides and the homogeneity of materials reduced to large extent [21]. Non-cleavage of the M-O-M' bond even upon hydrolysis followed by dehydration, makes homogenous oxides of high surface area mixed metal oxides nanoparticles. Consequently, the metallic oxoalkoxide complexes are considered as suitable precursors over others such as metal nitrate, acetate, monodispersed metal hydrous oxides, mainly due to the ease of their purification, solubility in organic solvents, volatility and their extremely facile hydrolizability.

*Corresponding author. E-mail: dhullrajesh79@gmail.com 
The careful handling is required to avoid phase segregation problem during preparation, substitution reaction and hydrolysis of such complexes. Many metal alkoxides thermally decompose in the range $\sim 100-300{ }^{\circ} \mathrm{C}$. Depending on process conditions, the thermolysis can afford nanosized powders of oxide or metallic phases. When certain specifications such as homogeneity, high purity, narrow particle-size distribution, fabrication of functional materials intended for aircraft, space, electronic fields, and chemical industry, individual oxides, their solid solutions, complex oxides, powders of metals and alloys active towards sintering, the solgel process is probably the best approach [22]. Hence, hydrothermally assisted sol-gel method is used for the preparation of hydrolyzed product. This method is simple, low temperature process and cost effective route for the preparation of crystalline oxides in comparison to conventional sol-gel method which is expensive one. Due to high sensitivity of metal alkoxides to the moisture and inhomogeneous precipitation hydothermal assisted sol-gel method is preferred over conventional sol-gel processing [10-13]. The aim of this work is to prepare suitable precursors for mixed metal oxide powders from their metal alkoxides by hydrothermally assisted sol-gel process.

\section{EXPERIMENTAL}

\section{Instrumentation, reagents and general techniques}

The infrared spectra were recorded on a Perkin-Elmer 1710 FTIR spectrometer over the range of $4000-400 \mathrm{~cm}^{-1}$. The ${ }^{1} \mathrm{H},{ }^{13} \mathrm{C},{ }^{27} \mathrm{Al},{ }^{11} \mathrm{~B}$ and ${ }^{119} \mathrm{Sn}$ NMR spectra were recorded in $\mathrm{CDCl}_{3}$ on Bruker Avance II 400 NMR spectrometer. TG study was made on Diamond TG/DTA PerkinElmer instrument. Elemental analyses were carried out by PerkinElmer 2400 II series CHNS/O Analyzer. The molecular weight data were determined by the cryoscopic method using a Beckman thermometer.

All the manipulations were carried out under dry nitrogen to exclude the moisture throughout the present investigations. Solvents and reagents used were purified and dried by standard methods [23-27]. Tin diacetate [ $\left.\mathrm{Sn}(\mathrm{OAc})_{2}\right]$ (Aldrich), aluminium isopropoxide $\left[\mathrm{Al}\left(\mathrm{OPr}^{\mathrm{i}}\right)_{3}\right]$ (Aldrich) and triisopropyl borate $\mathrm{B}\left(\mathrm{OPr}^{\mathrm{i}}\right)_{3}$ (Aldrich) were used as received. Benzene was purchased from Ranbaxy, kept in presence of sodium wire for two days and distilled twice before use to ensure the complete removal of moisture. Methyl salicylate, ethyl salicylate, phenyl salicylate and phenylethyl salicylate were purchased from Aldrich and used as received.

The isopropoxy groups in the $\mu$-oxoisopropoxide compound and liberated isopropanol formed in preparation of salicylate derivatives were estimated oxidimetrically [28]. Tin and aluminium were analyzed gravimetrically and boron was estimated volumetrically [26]. Further, spectral study of tin, aluminium and boron has also been carried out to find out their respective coordination number in order to elucidate the structure of $\mu$-oxo compounds. The salicylate derivatives of $\mu$-oxo compound $\left[\mathrm{SnO}_{2} \mathrm{AlB}\left(\mathrm{OPr}^{\mathrm{i}}\right)_{4}\right]$ were decomposed in conc. $\mathrm{HCl}$ and extracted in dilute $\mathrm{HCl}$, tin was precipitated as sulfide ( $\mathrm{pH} 5-6$ ), filtered and estimated as $\mathrm{SnO}_{2}$ [26]. The $\mathrm{H}_{2} \mathrm{~S}$ was boiled off completely from the filtrate and aluminium was estimated as aluminium oxinate [26].

Synthesis of $\left[\mathrm{SnO}_{2} \mathrm{AlB}\left(\mathrm{OPr}^{i}\right)_{4}\right]$ and its derivatives with salicylates

$\left[\mathrm{SnO}_{2} \mathrm{AlB}\left(\mathrm{OPr}^{\mathrm{i}}\right)_{4}\right]$ was prepared in laboratory by reported methods [29-31]. The complex $\left[\mathrm{SnO}_{2} \mathrm{AlB}\left(\mathrm{OPr}^{\mathrm{i}}\right)_{4}\right](0.434 \mathrm{~g}, 1.021 \mathrm{mmol})$ and methyl salicylate $(0.155 \mathrm{~g}, 1.021 \mathrm{mmol})$ were refluxed in benzene $(\sim 50 \mathrm{~mL})$ for $4 \mathrm{~h}$ at $\sim 100{ }^{\circ} \mathrm{C}$ in a flask connected to short distillation column. The liberated isopropanol was collected continuously at $72-78^{\circ} \mathrm{C}$ as a binary azeotrope of isoproponol-benzene [24]. The isopropanol in azeotrope was estimated oxidimetrically to check the completion of the reaction. The excess of the solvent was then removed at reduced 
pressure $\left(45{ }^{\circ} \mathrm{C} / 1 \mathrm{~mm} \mathrm{Hg}\right)$ yielding a yellowish brown solid product. Similar procedure was adopted for the preparation of other derivatives of $\left[\mathrm{SnO}_{2} \mathrm{AlB}\left(\mathrm{OPr}^{\mathrm{i}}\right)_{4}\right]$ with Salicylates (HRSAL), i.e. methyl salicylate $\left(\mathrm{HRSAL}^{1}\right)$, ethyl salicylate $\left(\mathrm{HRSAL}^{2}\right)$, phenyl salicylate $\left(\mathrm{HRSAL}^{3}\right)$, phenyl ethyl salicylate (HRSAL ${ }^{4}$ ) in stiochiometric ratio of $1: 1$ and 1:2 molar ratios. The details along with analytical data are given in Table 1 .

Table 1. Analytical and physical data of studied compounds.

\begin{tabular}{|c|c|c|c|c|c|c|c|c|c|c|c|}
\hline \multirow{2}{*}{$\begin{array}{c}\text { S. } \\
\text { No. }\end{array}$} & \multirow{2}{*}{$\begin{array}{l}\text { Compound } \\
\mathrm{g}(\mathrm{mmol})\end{array}$} & \multirow{2}{*}{$\begin{array}{l}\text { Ligand } \\
\mathrm{g}(\mathrm{mmol})\end{array}$} & \multirow{2}{*}{\begin{tabular}{|l}
$\begin{array}{l}\text { Reflu- } \\
\mathrm{x} \text { time } \\
(\mathrm{h})\end{array}$ \\
\end{tabular}} & \multirow{2}{*}{$\begin{array}{l}\text { Product } \\
\mathrm{g}(\%)\end{array}$} & \multicolumn{7}{|c|}{ Anal. Found (calcd.) } \\
\hline & & & & & $\begin{array}{l}\mathrm{OPr}^{\mathrm{i}} \\
(\mathrm{g})\end{array}$ & $\operatorname{Sn}(\%)$ & $\begin{array}{l}\mathrm{Al} \\
(\%) \\
\end{array}$ & $\begin{array}{c}\mathrm{B} \\
(\%) \\
\end{array}$ & $\begin{array}{c}\mathrm{C} \\
(\%)\end{array}$ & $\begin{array}{c}\mathrm{H} \\
(\%)\end{array}$ & $\begin{array}{c}\mathrm{O} \\
(\%)\end{array}$ \\
\hline 1 & & & 6 & & & & \begin{tabular}{|c|}
5.25 \\
$(5.22)$ \\
\end{tabular} & $\begin{array}{c}2.10 \\
(2.13) \\
\end{array}$ & & \begin{tabular}{|c|}
5.32 \\
$(5.41)$ \\
\end{tabular} & \\
\hline 2 & $\begin{array}{r}{[\mathrm{SnO}} \\
0.3 \\
\end{array}$ & $\mathrm{HP}$ & $11^{1 / 2}$ & $\begin{array}{r}\mathrm{nO}_{2} \mathrm{AlB} \\
0 .\end{array}$ & & & $\begin{array}{r}4.36 \\
(4.43) \\
\end{array}$ & $\begin{array}{l}1.79 \\
(1.81)\end{array}$ & \begin{tabular}{|l|}
43.29 \\
$(43.35)$ \\
\end{tabular} & \begin{tabular}{|c|}
4.54 \\
$(4.60)$ \\
\end{tabular} & \\
\hline 3 & $\begin{array}{r}\mathrm{SnO} \\
0.4 \\
\end{array}$ & $\mathrm{HR}$ & $61 / 2$ & $\left.\mathrm{SAI}^{2}\right)$ & $\begin{array}{c}0.08 \\
(0.07)\end{array}$ & $\begin{array}{c}22.31 \\
(22.45)\end{array}$ & \begin{tabular}{|c|}
5.05 \\
$(5.09)$ \\
\end{tabular} & \begin{tabular}{|c|}
2.03 \\
$(2.08)$ \\
\end{tabular} & \begin{tabular}{|c|}
40.63 \\
$(40.75)$ \\
\end{tabular} & \begin{tabular}{|c|}
5.57 \\
$(5.66)$ \\
\end{tabular} & \\
\hline 4 & $\begin{array}{r}{[\mathrm{SnO}} \\
0.3 \\
\end{array}$ & & $12^{1 / 2}$ & $\begin{array}{r}\mathrm{nO}_{2} \mathrm{AlB}( \\
0.3 \\
\end{array}$ & & & $\begin{array}{c}4.19 \\
(4.25) \\
\end{array}$ & \begin{tabular}{|c|}
1.69 \\
$(1.73)$ \\
\end{tabular} & \begin{tabular}{|c|}
45.23 \\
$(45.35)$ \\
\end{tabular} & \begin{tabular}{|c|}
4.93 \\
$(5.04)$ \\
\end{tabular} & \\
\hline 5 & $\begin{array}{r}\mathrm{SnO} \\
0.4\end{array}$ & $\mathrm{HR}$ & $5 \frac{1 / 2}{2}$ & $\begin{array}{r}\mathrm{SnO}_{2} \mathrm{All} \\
0 \\
\end{array}$ & & & $\begin{array}{c}4.58 \\
(4.65) \\
\end{array}$ & \begin{tabular}{|c|}
1.87 \\
$(1.90)$ \\
\end{tabular} & & \begin{tabular}{|c|}
5.08 \\
$(5.17)$ \\
\end{tabular} & \\
\hline 6 & $\begin{array}{r}\mathrm{SnO} \\
0.3 \\
\end{array}$ & $\begin{array}{r}\mathrm{HR} \\
0.35 \\
\end{array}$ & 11 & $\begin{array}{r}\mathrm{SnO}_{2} \mathrm{AlB} \\
0.4 \\
\end{array}$ & $\begin{array}{l}0.03 \\
(0.10)\end{array}$ & \begin{tabular}{|c|}
16.05 \\
$(16.19)$
\end{tabular} & \begin{tabular}{|c|}
3.65 \\
$(3.67)$ \\
\end{tabular} & $\begin{array}{c}1.49 \\
(1.50)\end{array}$ & \begin{tabular}{|l|}
52.13 \\
$(52.24)$ \\
\end{tabular} & \begin{tabular}{|c|}
4.24 \\
$(4.35)$ \\
\end{tabular} & \\
\hline 7 & $\begin{array}{r}{\left[\mathrm{SnO}_{2} \mathrm{~A}\right.} \\
0.440 \\
\end{array}$ & $\begin{array}{l}\text { HRSAL }^{4} \\
.252(1.035\end{array}$ & 6 & $\begin{array}{r}\mathrm{SnO}_{2} \mathrm{AlB}(\mathrm{OPr} \\
0.514(8 \\
\end{array}$ & $\begin{array}{c}0.04 \\
(0.06)\end{array}$ & $\begin{array}{c}19.47 \\
(19.54)\end{array}$ & $\begin{array}{c}4.39 \\
(4.43) \\
\end{array}$ & \begin{tabular}{|c|}
1.78 \\
$(1.81)$ \\
\end{tabular} & \begin{tabular}{|c|}
47.18 \\
$(47.29)$ \\
\end{tabular} & \begin{tabular}{|c|}
5.52 \\
$(5.58)$ \\
\end{tabular} & $\begin{array}{c}20.89 \\
(21.02) \\
\end{array}$ \\
\hline 8 & $\begin{array}{c}\mathrm{SnO}_{2} \mathrm{AlB}\left(\mathrm{OPr}^{\mathrm{i}}\right)_{4} \\
0.358(0.842)\end{array}$ & $\begin{array}{l}\text { HRSAL }^{4} \\
.410(1.684) \\
\end{array}$ & 12 & 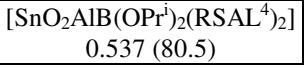 & $\begin{array}{c}0.05 \\
(0.10) \\
\end{array}$ & $\begin{array}{c}14.90 \\
(15)\end{array}$ & $\begin{array}{c}3.33 \\
(3.40) \\
\end{array}$ & $\begin{array}{r}1.35 \\
(1.39 \\
\end{array}$ & \begin{tabular}{|c|}
54.28 \\
$(54.47)$ \\
\end{tabular} & \begin{tabular}{|c|}
4.97 \\
$(5.04)$ \\
\end{tabular} & $\begin{array}{c}20.03 \\
(20.17) \\
\end{array}$ \\
\hline
\end{tabular}

The hydrolyzed product of salicylates of $\mu$-oxo compound was obtained by hydrothermally assisted sol-gel processing. For the hydrothermally assisted sol-gel processing, salicylate derivatives were diluted 20 times by weight with isopropanol, the mixture was loaded into a glass container and transferred into a $300 \mathrm{~mL}$ stainless steel autoclave. Dilution of salicylate derivatives and their transfer into autoclave was performed in moisture-free atmosphere to prevent their hydrolysis before introducing into a hydrothermal chamber. The gap between glass container and chamber was filled with $40 \mathrm{~mL}$ of distilled water and then the autoclave was tightly closed. The chamber was heated $120{ }^{\circ} \mathrm{C}$ for five hours, the autoclave is cooled and the product was filtered off and dried overnight at $100{ }^{\circ} \mathrm{C}$.

\section{RESULTS AND DISCUSSION}

Many reactions of $\left[\mathrm{SnO}_{2} \mathrm{AlB}\left(\mathrm{OPr}^{\mathrm{i}}\right)_{4}\right]$ with Salicylates (HRSAL), i.e. methyl salicylate $\left(\mathrm{HRSAL}^{1}\right)$, ethyl salicylate $\left(\mathrm{HRSAL}^{2}\right)$, phenyl salicylate $\left(\mathrm{HRSAL}^{3}\right)$, phenyl ethyl salicylate $\left(\mathrm{HRSAL}^{4}\right)$ were performed in different molar ratios in refluxing benzene, in which the products of type $\left[\mathrm{SnO}_{2} \mathrm{AlB}\left(\mathrm{OPr}^{\mathrm{i}}\right)_{3}(\mathrm{RSAL})\right],\left[\mathrm{Bu}_{2} \mathrm{SnO}_{2} \mathrm{Ti}_{2}\left(\mathrm{OPr}^{\mathrm{i}}\right)_{2}(\mathrm{RSAL})_{2}\right]$ were obtained. The general reaction can be given as follows:

$$
\left[\mathrm{SnO}_{2} \mathrm{AlB}\left(\mathrm{OPr}^{\mathrm{i}}\right)_{4}\right]+\mathrm{nHRSAL} \underset{\mathrm{Nefluxing} \text { benzene }}{\longrightarrow}\left[\mathrm{SnO}_{2} \mathrm{AlB}\left(\mathrm{OPr}^{\mathrm{i}}\right)_{4-\mathrm{n}}(\mathrm{RSAL})_{\mathrm{n}}\right]+\mathrm{nPr}^{\mathrm{i}} \mathrm{OH}
$$

where $\mathrm{n}=1-2$ and $\mathrm{HRSAL}=$ methyl/ethyl/phenyl/phenyl ethyl salicylates

The isopropanol liberated during the course of reaction was collected azeotropically (isopropanol-benzene) and estimated oxidimetrically to check the progress of the reaction and it was observed that only two out of four of isopropoxy groups of $\left[\mathrm{SnO}_{2} \mathrm{AlB}\left(\mathrm{OPr}^{\mathrm{i}}\right)_{4}\right]$ could be replaced with salicylate. Further replacement of third and fourth isopropoxy groups could not 
be achieved even with an excess of ligand (salicylate) and prolonged refluxing time (approx. 20 hours). All derivatives were found to be yellowish brown solid product, soluble in common organic solvents such as benzene, chloroform and hexane, low susceptible to hydrolysis and decomposed on heating $\left(\sim 180^{\circ} \mathrm{C}\right)$.

IR spectra

The IR spectra of the 1:1 salicylate derivatives of $\left[\mathrm{SnO}_{2} \mathrm{AlB}\left(\mathrm{OPr}^{\mathrm{i}}\right)_{4}\right]$ show absorption bands in the region $1365-1361 \mathrm{~cm}^{-1}$ and $1165-1150 \mathrm{~cm}^{-1}$ are the characterstics of $\mathrm{gem}$-dimethyl portion and combination band $v\left(\mathrm{C}-\mathrm{O}+\mathrm{OPr}^{\mathrm{i}}\right)$ of the bridging and terminal isopropoxy group respectively [28]. No peak is observed centered at $1165-1150 \mathrm{~cm}^{-1}$ in the spectrum of $1: 2$ salicylate derivatives indicates the absence of terminal isopropoxy group. A band appeared at approximately $950 \mathrm{~cm}^{-1}$ is due to $v(\mathrm{C}-\mathrm{O})$ stretching of bridging and terminal isopropoxy group. No bands other than 1:2 derivatives are observed in spectra of compounds formed by reactions of 1:3 and 1:4 molar ratios of $\mu$-oxo compound and salicylates states the non-replacement of bridging isopropoxy groups by salicylates. The $v(\mathrm{O}-\mathrm{H})$ band occurring in the region $~ 3400-$ $3100 \mathrm{~cm}^{-1}$ in the salicylates disappears completely in the derivatives, indicating the deprotonation of salicylates ligands. In salicylates, the band appearing at $\sim 1645 \mathrm{~cm}^{-1}$ due to $v(C=O)$ shows a downward shift of $15-25 \mathrm{~cm}^{-1}$ in the derivatives, indicating the coordination of the carbonyl oxygen of the salicylate to the metal atom. A strong band observed at $\sim 1250 \mathrm{~cm}^{-1}$ in salicylates due to phenolic $v(\mathrm{C}-\mathrm{O})$ vibrations is shifted $10-20 \mathrm{~cm}^{-1}$ higher in the derivatives indicating bond formation of phenolic oxygen of salicylate to the metal atom [28]. A number of peaks are observed in the region $700-400 \mathrm{~cm}^{-1}$ due to $\mathrm{M}-\mathrm{O}$ stretching vibrations in $\mu$-oxo compounds which is difficult to assign exactly due to the overlapping of bands in this region [32]. The detail of IR spectra is given in Table 2 .

Table 2. Important bands of FTIR spectra.

\begin{tabular}{|c|c|c|}
\hline S.N. & Compound & FT-IR frequencies $\left(\mathrm{cm}^{-1}\right)$ \\
\hline 1. & {$\left[\mathrm{SnO}_{2} \mathrm{AlB}\left(\mathrm{OPr}^{\mathrm{i}}\right)_{3}\left(\mathrm{RSAL}^{1}\right)\right]$} & $\begin{array}{l}2968 \mathrm{~s} \text { and } 2853 \mathrm{~m}, v(\mathrm{C}-\mathrm{H}) ; 1620 \mathrm{~s}, v(\mathrm{C}=\mathrm{O}) ; 1260 \mathrm{~m}, \\
v(\mathrm{C}-\mathrm{O}) \text { phenolic; } 1361 \mathrm{~s}, \mathrm{v}\left(\mathrm{C}-\mathrm{O}+\mathrm{OPr}^{\mathrm{i}}\right) \text { bridging; } 1150 \mathrm{~s}, v\left(\mathrm{C}-\mathrm{O}+\mathrm{OPr}^{\mathrm{i}}\right) \\
\text { terminal; } 952 \mathrm{~m}, v(\mathrm{C}-\mathrm{O}) ; 698 \mathrm{~m}, 593 \mathrm{~m}, 476 \mathrm{w}, 425 \mathrm{~m}, 433 \mathrm{w}, \mathrm{v}(\mathrm{M}-\mathrm{O})\end{array}$ \\
\hline 2. & {$\left[\mathrm{SnO}_{2} \mathrm{AlB}\left(\mathrm{OPr}^{\mathrm{i}}\right)_{2}\left(\mathrm{RSAL}^{1}\right)_{2}\right]$} & $\begin{array}{l}2995 \mathrm{~s} \text { and } 2864 \mathrm{~m}, \mathrm{v}(\mathrm{C}-\mathrm{H}) ; 1627 \mathrm{~s}, v(\mathrm{C}=\mathrm{O}) ; 1266 \mathrm{~m}, v(\mathrm{C}-\mathrm{O}) \text { phenolic; } \\
1365 \mathrm{~s}, v(\mathrm{C}-\mathrm{O}+\mathrm{OPr}) \text { bridging; } 950 \mathrm{~m}, v(\mathrm{C}-\mathrm{O}) ; 700 \mathrm{~m}, 593 \mathrm{~m}, 486 \mathrm{w} \\
428 \mathrm{~m}, 439 \mathrm{w}, \mathrm{v}(\mathrm{M}-\mathrm{O})\end{array}$ \\
\hline 3. & {$\left[\mathrm{SnO}_{2} \mathrm{AlB}\left(\mathrm{OPr}^{\mathrm{i}}\right)_{3}\left(\mathrm{RSAL}^{2}\right)\right]$} & $\begin{array}{l}2985 \mathrm{~s} \text { and } 2851 \mathrm{~m}, v(\mathrm{C}-\mathrm{H}) ; 1622 \mathrm{~s}, v(\mathrm{C}=\mathrm{O}) ; 1261 \mathrm{~m}, v(\mathrm{C}-\mathrm{O}) \text { phenolic; } \\
1360 \mathrm{~s}, v\left(\mathrm{C}-\mathrm{O}+\mathrm{OPr}^{\mathrm{i}}\right) \text { bridging; } 1158 \mathrm{~s}, v\left(\mathrm{C}-\mathrm{O}+\mathrm{OPr}^{\mathrm{r}}\right) \text { terminal; } 950 \mathrm{~m} \\
\mathrm{v}(\mathrm{C}-\mathrm{O}) ; 695 \mathrm{~m}, 589 \mathrm{~m}, 485 \mathrm{w}, 424 \mathrm{~m}, 429 \mathrm{w}, v(\mathrm{M}-\mathrm{O})\end{array}$ \\
\hline 4. & {$\left[\mathrm{SnO}_{2} \mathrm{AlB}\left(\mathrm{OPr}^{\mathrm{i}}\right)_{2}\left(\mathrm{RSAL}^{2}\right)_{2}\right]$} & $\begin{array}{l}2997 \mathrm{~s} \text { and } 2862 \mathrm{~m}, \mathrm{v}(\mathrm{C}-\mathrm{H}) ; 1625 \mathrm{~s}, v(\mathrm{C}=\mathrm{O}) ; 1269 \mathrm{~m}, \mathrm{v}(\mathrm{C}-\mathrm{O}) \text { phenolic; } \\
\left.1364 \mathrm{~s}, v(\mathrm{C}-\mathrm{O}+\mathrm{OPr})^{\mathrm{i}}\right) \text { bridging; } 953 \mathrm{~m}, \mathrm{v}(\mathrm{C}-\mathrm{O}) ; 699 \mathrm{~m}, 597 \mathrm{~m}, 488 \mathrm{w} \\
432 \mathrm{~m}, 446 \mathrm{w}, \mathrm{v}(\mathrm{M}-\mathrm{O})\end{array}$ \\
\hline 5. & {$\left[\mathrm{SnO}_{2} \mathrm{AlB}\left(\mathrm{OPr}^{\mathrm{i}}\right)_{3}\left(\mathrm{RSAL}^{3}\right)\right]$} & $\begin{array}{l}2965 \mathrm{~s} \text { and } 2855 \mathrm{~m}, v(\mathrm{C}-\mathrm{H}) ; 1625 \mathrm{~s}, \mathrm{v}(\mathrm{C}=\mathrm{O}) ; 1263 \mathrm{~m}, \mathrm{v}(\mathrm{C}-\mathrm{O}) \text { phenolic; } \\
1362 \mathrm{~s}, v\left(\mathrm{C}-\mathrm{O}+\mathrm{OPr}^{\mathrm{i}}\right) \text { bridging; } 1162 \mathrm{~s}, \mathrm{v}\left(\mathrm{C}-\mathrm{O}+\mathrm{OPr}^{\mathrm{r}}\right) \text { terminal; } 948 \mathrm{~m} \text {, } \\
v(\mathrm{C}-\mathrm{O}) ; 697 \mathrm{~m}, 598 \mathrm{~m}, 487 \mathrm{w}, 428 \mathrm{~m}, 435 \mathrm{w}, \mathrm{v}(\mathrm{M}-\mathrm{O})\end{array}$ \\
\hline 6. & {$\left[\mathrm{SnO}_{2} \mathrm{AlB}\left(\mathrm{OPr}^{\mathrm{i}}\right)_{2}\left(\mathrm{RSAL}^{3}\right)_{2}\right]$} & $\begin{array}{l}2989 \mathrm{~s} \text { and } 2866 \mathrm{~m}, v(\mathrm{C}-\mathrm{H}) ; 1630 \mathrm{~s}, v(\mathrm{C}=\mathrm{O}) ; 1268 \mathrm{~m}, v(\mathrm{C}-\mathrm{O}) \text { phenolic; } \\
1365 \mathrm{~s}, v\left(\mathrm{C}-\mathrm{O}+\mathrm{OPr} \mathrm{Pr}^{\mathrm{i}}\right) \text { bridging; } 950 \mathrm{~m}, v(\mathrm{C}-\mathrm{O}) ; 701 \mathrm{~m}, 593 \mathrm{~m}, 489 \mathrm{w}, \\
437 \mathrm{~m}, 449 \mathrm{w}, \mathrm{v}(\mathrm{M}-\mathrm{O})\end{array}$ \\
\hline 7. & {$\left[\mathrm{SnO}_{2} \mathrm{AlB}\left(\mathrm{OPr}^{\mathrm{i}}\right)_{3}\left(\mathrm{RSAL}^{4}\right)\right]$} & $\begin{array}{l}2989 \mathrm{~s} \text { and } 2858 \mathrm{~m}, v(\mathrm{C}-\mathrm{H}) ; 1629 \mathrm{~s}, \mathrm{v}(\mathrm{C}=\mathrm{O}) ; 1270 \mathrm{~m}, \mathrm{v}(\mathrm{C}-\mathrm{O}) \text { phenolic; } \\
1360 \mathrm{~s}, v\left(\mathrm{C}-\mathrm{O}+\mathrm{OPr}^{\mathrm{i}}\right) \text { bridging; } 1165 \mathrm{~s}, \mathrm{v}\left(\mathrm{C}-\mathrm{O}+\mathrm{OPr}^{\mathrm{r}}\right) \text { terminal; } 945 \mathrm{~m} \text {, } \\
\mathrm{v}(\mathrm{C}-\mathrm{O}) ; 699 \mathrm{~m}, 581 \mathrm{~m}, 498 \mathrm{w}, 433 \mathrm{~m}, 438 \mathrm{w} ; \mathrm{v}(\mathrm{M}-\mathrm{O})\end{array}$ \\
\hline 8. & {$\left[\mathrm{SnO}_{2} \mathrm{AlB}\left(\mathrm{OPr}^{\mathrm{i}}\right)_{2}\left(\mathrm{RSAL}^{4}\right)_{2}\right]$} & $\begin{array}{l}2997 \mathrm{~s} \text { and } 2859 \mathrm{~m}, \mathrm{v}(\mathrm{C}-\mathrm{H}) ; 1629 \mathrm{~s}, v(\mathrm{C}=\mathrm{O}) ; 1269 \mathrm{~m}, \mathrm{v}(\mathrm{C}-\mathrm{O}) \text { phenolic; } \\
1364 \mathrm{~s}, \mathrm{v}(\mathrm{C}-\mathrm{O}+\mathrm{OPr}) \text { bridging; } 955 \mathrm{~m}, \mathrm{v}(\mathrm{C}-\mathrm{O}) ; 699 \mathrm{~m}, 594 \mathrm{~m}, 479 \mathrm{w} \\
435 \mathrm{~m}, 452 \mathrm{w}, \mathrm{v}(\mathrm{M}-\mathrm{O})\end{array}$ \\
\hline
\end{tabular}

Bull. Chem. Soc. Ethiop. 2013, 27(3) 


\section{NMR spectral studies}

${ }^{l} H$ NMR. ${ }^{1} \mathrm{H}$ NMR spectra of all the salicylate derivatives of $\left[\mathrm{SnO}_{2} \mathrm{AlB}\left(\mathrm{OPr}^{\mathrm{i}}\right)_{4}\right]$ show broad multiplet centered between $\delta$ 0.8-1.2 ppm due to the intermixing of methyl protons of isopropoxy groups [25]. A broad multiplet centered at $\delta 4.0-4.2 \mathrm{ppm}$ is due to the methine proton of isopropoxy groups in the spectra of all derivatives. The bridging isopropoxy groups are not substituted by salicylates because the same peaks of 1:2 derivatives are observed in the spectra taken for compounds formed by reactions of 1:3 and 1:4 molar ratios of parent compound and salicylates. A number of signals observed between $\delta 6.4-7.5 \mathrm{ppm}$ are observed for phenyl ring protons in phenyl salicylate derivatives and phenyl ethyl protons in phenyl ethyl salicylate derivatives. The phenolic proton peak $(\mathrm{O}-\mathrm{H})$ of salicylates at $\delta 9.2 \mathrm{ppm}$ is found deficient in their derivatives indicates the deprotonation of phenolic group (Table 3 ).

Table 3. Important signals of ${ }^{1} \mathrm{H}$ and ${ }^{13} \mathrm{C}$ NMR spectra.

\begin{tabular}{|c|c|c|c|}
\hline S.N. & Compound & ${ }^{1} \mathrm{H}$ NMR $(\delta$ ppm $)$ & ${ }^{13} \mathrm{C}$ NMR $(\delta \mathrm{ppm})$ \\
\hline 1. & {$\left[\mathrm{SnO}_{2} \mathrm{AlB}\left(\mathrm{OPr}^{\mathrm{i}}\right)_{3}\left(\mathrm{RSAL}^{1}\right)\right]$} & $\begin{array}{l}1.0\left(\mathrm{~d}, \mathrm{CH}_{3}\right), 1.2\left(\mathrm{~d}, \mathrm{CH}_{3}\right), \\
4.1(\mathrm{~m}, \mathrm{CH}), 2.6\left(\mathrm{~s}, \mathrm{CH}_{3}\right), \\
7.1-7.3(\mathrm{~m}, \mathrm{CH})\end{array}$ & $\begin{array}{l}27.4\left(\mathrm{~s}, \mathrm{CH}_{3}\right), 27.9\left(\mathrm{~s}, \mathrm{CH}_{3}\right), 62.6(\mathrm{~s}, \mathrm{CH}), \\
66.2(\mathrm{~s}, \mathrm{CH}), 168.2(\mathrm{~s}, \mathrm{Ar} \mathrm{CH}), 129.5-138.6 \\
(\mathrm{~m}, \mathrm{Ar} \mathrm{CH}), 51.8\left(\mathrm{~s}, \mathrm{CH}_{3}\right), 180.2(\mathrm{~s}, \mathrm{C}=\mathrm{O})\end{array}$ \\
\hline 2. & {$\left[\mathrm{SnO}_{2} \mathrm{AlB}\left(\mathrm{OPr}^{\mathrm{i}}\right)_{2}\left(\mathrm{RSAL}^{1}\right)_{2}\right]$} & $\begin{array}{l}0.8\left(\mathrm{~d}, \mathrm{CH}_{3}\right), 1.1\left(\mathrm{~d}, \mathrm{CH}_{3}\right) \\
4.0(\mathrm{~m}, \mathrm{CH}), 2.4\left(\mathrm{~s}, \mathrm{CH}_{3}\right) \\
6.9-7.2(\mathrm{~m}, \mathrm{CH})\end{array}$ & $\begin{array}{l}27.2\left(\mathrm{~s}, \mathrm{CH}_{3}\right), 27.7\left(\mathrm{~s}, \mathrm{CH}_{3}\right), 62.1(\mathrm{~s}, \mathrm{CH}) \\
65.8(\mathrm{~s}, \mathrm{CH}), 167.7(\mathrm{~s}, \mathrm{Ar} \mathrm{CH}), 129.0-136.3 \\
(\mathrm{~m}, \mathrm{ArCH}), 51.5\left(\mathrm{~s}, \mathrm{CH}_{3}\right), 178.1(\mathrm{~s}, \mathrm{C}=\mathrm{O})\end{array}$ \\
\hline 3. & {$\left[\mathrm{SnO}_{2} \mathrm{AlB}\left(\mathrm{OPr}^{\mathrm{i}}\right)_{3}\left(\mathrm{RSAL}^{2}\right)\right]$} & $\begin{array}{l}1.1\left(\mathrm{~d}, \mathrm{CH}_{3}\right), 1.2\left(\mathrm{~d}, \mathrm{CH}_{3}\right) \\
4.2(\mathrm{~m}, \mathrm{CH}), 2.4\left(\mathrm{~s}, \mathrm{CH}_{3}\right), \\
\text { 7.2-7.5 (m, CH) }\end{array}$ & $\begin{array}{l}27.5\left(\mathrm{~s}, \mathrm{CH}_{3}\right), 28.2\left(\mathrm{~s}, \mathrm{CH}_{3}\right), 62.5(\mathrm{~s}, \mathrm{CH}) \\
66.5(\mathrm{~s}, \mathrm{CH}), 167.5(\mathrm{~s}, \mathrm{Ar} \mathrm{CH}), 128.4-139.7 \\
(\mathrm{~m}, \mathrm{ArCH}), 52.4\left(\mathrm{~s}, \mathrm{CH}_{3}\right), 179.5(\mathrm{~s}, \mathrm{C}=\mathrm{O})\end{array}$ \\
\hline 4. & {$\left[\mathrm{SnO}_{2} \mathrm{AlB}\left(\mathrm{OPr}^{\mathrm{i}}\right)_{2}\left(\mathrm{RSAL}^{2}\right)_{2}\right]$} & $\begin{array}{l}0.9\left(\mathrm{~d}, \mathrm{CH}_{3}\right), 1.1\left(\mathrm{~d}, \mathrm{CH}_{3}\right), \\
4.2(\mathrm{~m}, \mathrm{CH}), 2.3\left(\mathrm{~s}, \mathrm{CH}_{3}\right), \\
6.8-7.3(\mathrm{~m}, \mathrm{CH})\end{array}$ & $\begin{array}{l}27.1\left(\mathrm{~s}, \mathrm{CH}_{3}\right), 27.8\left(\mathrm{~s}, \mathrm{CH}_{3}\right), 62.4(\mathrm{~s}, \mathrm{CH}) \\
65.7(\mathrm{~s}, \mathrm{CH}), 167.3(\mathrm{~s}, \mathrm{Ar} \mathrm{CH}), 128.3-137.9 \\
(\mathrm{~m}, \mathrm{Ar} \mathrm{CH}), 52.5\left(\mathrm{~s}, \mathrm{CH}_{3}\right), 175.9(\mathrm{~s}, \mathrm{C}=\mathrm{O})\end{array}$ \\
\hline 5. & {$\left[\mathrm{SnO}_{2} \mathrm{AlB}\left(\mathrm{OPr}^{\mathrm{i}}\right)_{3}\left(\mathrm{RSAL}^{3}\right)\right]$} & $\begin{array}{l}\left.\text { lo (d, } \mathrm{CH}_{3}\right), 1.2\left(\mathrm{~d}, \mathrm{CH}_{3}\right), \\
4.2(\mathrm{~m}, \mathrm{CH}), 2.4\left(\mathrm{~s}, \mathrm{CH}_{3}\right), \\
7.2-7.5(\mathrm{~m}, \mathrm{CH})\end{array}$ & $\begin{array}{l}27.5\left(\mathrm{~s}, \mathrm{CH}_{3}\right), 27.8\left(\mathrm{~s}, \mathrm{CH}_{3}\right), 62.7(\mathrm{~s}, \mathrm{CH}) \\
66.5(\mathrm{~s}, \mathrm{CH}), 168.2(\mathrm{~s}, \mathrm{Ar} \mathrm{CH}), 130.3-140.3 \\
(\mathrm{~m}, \mathrm{Ar} \mathrm{CH}), 50.7\left(\mathrm{~s}, \mathrm{CH}_{3}\right), 181.5(\mathrm{~s}, \mathrm{C}=\mathrm{O})\end{array}$ \\
\hline 6. & {$\left[\mathrm{SnO}_{2} \mathrm{AlB}\left(\mathrm{OPr}^{\mathrm{i}}\right)_{2}\left(\mathrm{RSAL}^{3}\right)_{2}\right]$} & $\begin{array}{l}0.8\left(\mathrm{~d}, \mathrm{CH}_{3}\right), 1.0\left(\mathrm{~d}, \mathrm{CH}_{3}\right), \\
4.1(\mathrm{~m}, \mathrm{CH}), 2.3\left(\mathrm{~s}, \mathrm{CH}_{3}\right), \\
6.9-7.1(\mathrm{~m}, \mathrm{CH})\end{array}$ & $\begin{array}{l}27.4\left(\mathrm{~s}, \mathrm{CH}_{3}\right), 28.1\left(\mathrm{~s}, \mathrm{CH}_{3}\right), 62.2(\mathrm{~s}, \mathrm{CH}) \\
66.7(\mathrm{~s}, \mathrm{CH}), 165.3(\mathrm{~s}, \mathrm{Ar} \mathrm{CH}), 128.4-138.7 \\
(\mathrm{~m}, \mathrm{Ar} \mathrm{CH}), 52.4\left(\mathrm{~s}, \mathrm{CH}_{3}\right), 176.6(\mathrm{~s}, \mathrm{C}=\mathrm{O})\end{array}$ \\
\hline 7. & {$\left[\mathrm{SnO}_{2} \mathrm{AlB}\left(\mathrm{OPr}^{\mathrm{i}}\right)_{3}\left(\mathrm{RSAL}^{4}\right)\right]$} & $\begin{array}{l}1.0\left(\mathrm{~d}, \mathrm{CH}_{3}\right), 1.2\left(\mathrm{~d}, \mathrm{CH}_{3}\right), \\
4.1(\mathrm{~m}, \mathrm{CH}), 2.2\left(\mathrm{~s}, \mathrm{CH}_{3}\right), \\
7.1-7.3(\mathrm{~m}, \mathrm{CH})\end{array}$ & $\begin{array}{l}26.7\left(\mathrm{~s}, \mathrm{CH}_{3}\right), 28.5\left(\mathrm{~s}, \mathrm{CH}_{3}\right), 62.6(\mathrm{~s}, \mathrm{CH}), \\
67.2(\mathrm{~s}, \mathrm{CH}), 169.1(\mathrm{~s}, \mathrm{Ar} \mathrm{CH}), 130.6-139.9 \\
(\mathrm{~m}, \mathrm{Ar} \mathrm{CH}), 53.5\left(\mathrm{~s}, \mathrm{CH}_{3}\right), 181.2(\mathrm{~s}, \mathrm{C}=\mathrm{O})\end{array}$ \\
\hline 8. & {$\left[\mathrm{SnO}_{2} \mathrm{AlB}\left(\mathrm{OPr}^{\mathrm{i}}\right)_{2}\left(\mathrm{RSAL}^{4}\right)_{2}\right]$} & $\begin{array}{l}0.9\left(\mathrm{~d}, \mathrm{CH}_{3}\right), 1.9\left(\mathrm{~d}, \mathrm{CH}_{3}\right) \\
4.0(\mathrm{~m}, \mathrm{CH}), 2.5\left(\mathrm{~s}, \mathrm{CH}_{3}\right) \\
6.4-7.0(\mathrm{~m}, \mathrm{CH})\end{array}$ & $\begin{array}{l}27.1\left(\mathrm{~s}, \mathrm{CH}_{3}\right), 28.4\left(\mathrm{~s}, \mathrm{CH}_{3}\right), 62.4(\mathrm{~s}, \mathrm{CH}) \\
66.4(\mathrm{~s}, \mathrm{CH}), 163.5(\mathrm{~s}, \mathrm{Ar} \mathrm{CH}), 128.5-138.5 \\
(\mathrm{~m}, \mathrm{Ar} \mathrm{CH}), 52.5\left(\mathrm{~s}, \mathrm{CH}_{3}\right), 179.9(\mathrm{~s}, \mathrm{C}=\mathrm{O})\end{array}$ \\
\hline
\end{tabular}

${ }^{13} \mathrm{C} N M R$. The ${ }^{13} \mathrm{C}$ NMR spectra of mono derivatives of $\left[\mathrm{SnO}_{2} \mathrm{AlB}\left(\mathrm{OPr}^{\mathrm{i}}\right)_{4}\right]$ shows two prominent peaks between $\delta$ 26.7-27.5 and $\delta$ 27.7-28.5 ppm assignable to the methyl carbon of terminal and interamolecularly bridged isopropoxy moiety and two different type of methine carbons of isopropoxy group is confirmed by the two signals observed centered at $\delta 62.1-62.7 \mathrm{ppm}$ and $\delta$ 65.8-67.2 ppm [33]. The spectra of 1:2 salicylate derivatives of $\mu$-oxoisopropoxide show one singlet for methyl and methane carbon of bridging isopropoxy groups. All signals of $\left[\mathrm{SnO}_{2} \mathrm{AlB}\left(\mathrm{OPr}^{\mathrm{i}}\right)_{2}(\mathrm{RSAL})_{2}\right]$ are experiential in the spectra taken for compounds formed by reactions of 1:3 and 1:4 molar ratios of $\mu$-oxo compound and salicylates confirms the nonreplacement of bridging isopropoxy groups by salicylates. The peaks observed in the region 8128.4-139.9 ppm are due to carbon atoms on benzene ring, however, the peak observed at about $8163.5-169.1 \mathrm{ppm}$ is due to ring carbon linked to the ester group and a peak observed at 
8175.9-181.5 ppm is due to carbon of the ester group (-COOR) [33]. The ${ }^{1} \mathrm{H}$ and ${ }^{13} \mathrm{C}$ NMR spectral data are given in Table 3.

${ }^{27} \mathrm{Al},{ }^{119} \mathrm{Sn}$, and ${ }^{11} B \mathrm{NMR}$. The ${ }^{27} \mathrm{Al}$ NMR spectra of mono derivatives $\left[\mathrm{SnO}_{2} \mathrm{AlB}(\mathrm{OPr})_{3}(\mathrm{RSAL})\right]$ show two singlet between $\delta$ 0.9-1.2 and $\delta 64.3-65.2 \mathrm{ppm}$ indicating octahedral and tetrahedral environment about the aluminium [34-35]. The spectra of $\left[\mathrm{SnO}_{2} \mathrm{AlB}\left(\mathrm{OPr}^{\mathrm{i}}\right)_{2}(\mathrm{RSAL})_{2}\right]$ demonstrate the only sinlet centered at $\delta 0.7-1.3 \mathrm{ppm}$ confirms the complete substitution of isopropoxy groups of aluminium by salicylates. A sharp signal centered at $\delta-489$ to $-467 \mathrm{ppm}$ in the ${ }^{119} \mathrm{Sn}$ NMR spectra of salicylate derivatives of $\left[\mathrm{SnO}_{2} \mathrm{AlB}\left(\mathrm{OPr}^{\mathrm{i}}\right)_{4}\right]$ indicates the tetrahedral environment surrounding the $\mathrm{Sn}(\mathrm{II})$ attributed to the polymeric nature of $\mu$-oxo compound [3637]. The ${ }^{11} B$ NMR study reveals the tetrahedral environment surrounding the $B$ in salicylate derivatives of parent compound [38]. The NMR spectral data are given in Table 4.

Table 4. Important peaks of ${ }^{27} \mathrm{Al},{ }^{119} \mathrm{Sn},{ }^{11} \mathrm{~B}$ NMR spectra.

\begin{tabular}{|c|c|c|c|c|}
\hline S. No. & Compound & ${ }^{27} \mathrm{Al}$ NMR $(\delta \mathrm{ppm})$ & ${ }^{119} \mathrm{Sn}$ NMR $(\delta \mathrm{ppm})$ & ${ }^{11} \mathrm{~B}$ NMR $(\delta \mathrm{ppm})$ \\
\hline 1. & {$\left[\mathrm{SnO}_{2} \mathrm{AlB}\left(\mathrm{OPr}^{\mathrm{i}}\right)_{3}\left(\mathrm{RSAL}^{1}\right)\right]$} & $\begin{array}{l}1.1[\mathrm{~s}, \mathrm{Al}(\mathrm{O})] \\
64.3[\mathrm{~s}, \mathrm{Al}(\mathrm{T})]\end{array}$ & $-489[\mathrm{~s}, \mathrm{Sn}(\mathrm{T})]$ & $3.87[\mathrm{~s}(\mathrm{~b}), \mathrm{B}(\mathrm{T})]$ \\
\hline 2. & {$\left[\mathrm{SnO}_{2} \mathrm{AlB}\left(\mathrm{OPr}^{\mathrm{i}}\right)_{2}\left(\mathrm{RSAL}^{1}\right)_{2}\right]$} & $0.8[\mathrm{~s}, \mathrm{Al}(\mathrm{O})]$ & $-477[\mathrm{~s}, \mathrm{Sn}(\mathrm{T})]$ & $3.75[\mathrm{~s}(\mathrm{~b}), \mathrm{B}(\mathrm{T})]$ \\
\hline 3. & {$\left[\mathrm{SnO}_{2} \mathrm{AlB}\left(\mathrm{OPr}^{\mathrm{i}}\right)_{3}\left(\mathrm{RSAL}^{2}\right)\right]$} & $\begin{array}{c}0.9[\mathrm{~s}, \mathrm{Al}(\mathrm{O})] \\
64.6[\mathrm{~s}, \mathrm{Al}(\mathrm{T})]\end{array}$ & $-485[\mathrm{~s}, \mathrm{Sn}(\mathrm{T})]$ & $3.79[\mathrm{~s}(\mathrm{~b}), \mathrm{B}(\mathrm{T})]$ \\
\hline 4. & {$\left[\mathrm{SnO}_{2} \mathrm{AlB}\left(\mathrm{OPr}^{\mathrm{i}}\right)_{2}\left(\mathrm{RSAL}^{2}\right)_{2}\right]$} & $0.7[\mathrm{~s}, \mathrm{Al}(\mathrm{O})]$ & $-471[\mathrm{~s}, \mathrm{Sn}(\mathrm{T})]$ & $3.59[\mathrm{~s}(\mathrm{~b}), \mathrm{B}(\mathrm{T})]$ \\
\hline 5. & {$\left[\mathrm{SnO}_{2} \mathrm{AlB}\left(\mathrm{OPr}^{\mathrm{i}}\right)_{3}\left(\mathrm{RSAL}^{3}\right)\right]$} & $\begin{array}{c}1.2[\mathrm{~s}, \mathrm{Al}(\mathrm{O})] \\
64.5[\mathrm{~s}, \mathrm{Al}(\mathrm{T})]\end{array}$ & $-482[\mathrm{~s}, \mathrm{Sn}(\mathrm{T})]$ & $3.64[\mathrm{~s}(\mathrm{~b}), \mathrm{B}(\mathrm{T})]$ \\
\hline 6. & {$\left[\mathrm{SnO}_{2} \mathrm{AlB}\left(\mathrm{OPr}^{\mathrm{i}}\right)_{2}\left(\mathrm{RSAL}^{3}\right)_{2}\right]$} & $0.8[\mathrm{~s}, \mathrm{Al}(\mathrm{O})]$ & $-470[\mathrm{~s}, \mathrm{Sn}(\mathrm{T})]$ & $3.47[\mathrm{~s}(\mathrm{~b}), \mathrm{B}(\mathrm{T})]$ \\
\hline 7. & {$\left[\mathrm{SnO}_{2} \mathrm{AlB}\left(\mathrm{OPr}^{\mathrm{i}}\right)_{3}\left(\mathrm{RSAL}^{4}\right)\right]$} & $\begin{array}{c}0.8[\mathrm{~s}, \mathrm{Al}(\mathrm{O})] \\
65.2[\mathrm{~s}, \mathrm{Al}(\mathrm{T})]\end{array}$ & $-487[\mathrm{~s}, \mathrm{Sn}(\mathrm{T})]$ & $3.65[\mathrm{~s}(\mathrm{~b}), \mathrm{B}(\mathrm{T})]$ \\
\hline 8. & {$\left[\mathrm{SnO}_{2} \mathrm{AlB}\left(\mathrm{OPr}^{\mathrm{i}}\right)_{2}\left(\mathrm{RSAL}^{4}\right)_{2}\right]$} & $1.3[\mathrm{~s}, \mathrm{Al}(\mathrm{O})]$ & $-479[\mathrm{~s}, \mathrm{Sn}(\mathrm{T})]$ & $3.23[\mathrm{~s}(\mathrm{~b}), \mathrm{B}(\mathrm{T})]$ \\
\hline
\end{tabular}

$(\mathrm{O})=$ Octahedral,$(\mathrm{T})=$ Tetrahedral .

\section{Thermal studies}

The thermal decomposition of salicylate derivatives of $\left[\mathrm{SnO}_{2} \mathrm{AlB}\left(\mathrm{OPr}^{\mathrm{i}}\right)_{4}\right]$ have been examined by thermogravimetric analysis under a flow of dry nitrogen, up to $800{ }^{\circ} \mathrm{C}$ at a heating rate of 20 ${ }^{\circ} \mathrm{C} / \mathrm{min}$. The TGA curve shows two stages of weight loss (Figure 1). The first and small step starts at $58.9^{\circ} \mathrm{C}$ and completed at $182{ }^{\circ} \mathrm{C}$ with a weight loss of $1.14 \%$ due to presence of moisture and fraction of solvent present, if any. The second and major one step starts at $182^{\circ} \mathrm{C}$ and is completed at $357^{\circ} \mathrm{C}$, resulting in a residue amounting to $11.25 \%$ of the initial weight, probably due to the decomposition of partially hydrolyzed $\mu$-oxo salicylate into metal $/$ mixed metal oxides suggesting the volatile nature of compound [39]. Similar thermograms observed for other salicylate derivatives of $\left[\mathrm{SnO}_{2} \mathrm{AlB}\left(\mathrm{OPr}^{\mathrm{i}}\right)_{4}\right]$.

The thermogravimetric analysis of various hydrolyzed product of different salicylate derivatives have been performed up to $800{ }^{\circ} \mathrm{C}$ at $10{ }^{\circ} \mathrm{C} / \mathrm{min}$. Thermograms of various hydrolysed salicylate derivatives studied as, the weight loss in stage (a) observed due to the traces of water and solvent present in hydrolyzed product of $\mu$-oxo compound. The major weight loss in stage (b) occurs probably due to the elimination of hydroxy groups and organic moieties present in the hydrolyzed product which is directly followed by last stage (c) ranging from 345 to $800{ }^{\circ} \mathrm{C}$, leaving a residue that is less than the calculated for mixed metal oxide and metal oxides $\left(\mathrm{SnAl}_{2} \mathrm{O}_{4}, \mathrm{SnO}\right.$ and $\left.\mathrm{B}_{2} \mathrm{O}_{3}\right)$. The TG of hydrolyzed product of mono derivative of $\mu$ oxo derivative with methyl salicylate is shown in Figure 2. The detailed study of thermograms of hydrolyzed product of various salicylate derivatives is summarized in Table 5. 
The molecular weight measurement carried out in dry benzene using cryoscopic method suggests the polymeric nature of all salicylate derivatives.

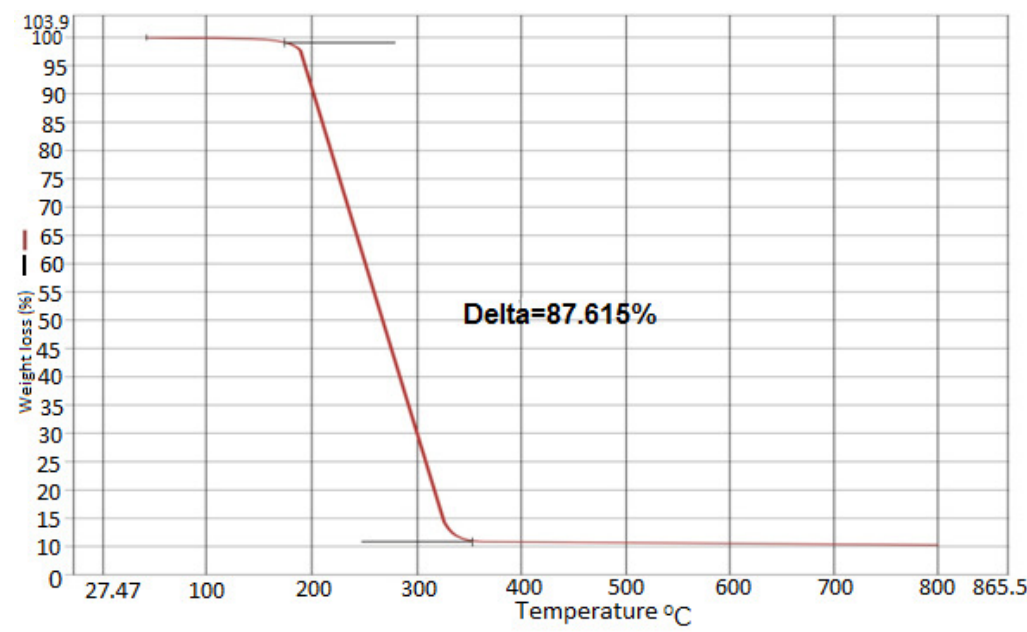

Figure 1. TG of $\left[\mathrm{SnO}_{2} \mathrm{AlB}\left(\mathrm{OPr}^{\mathrm{i}}\right)_{3}\left(\mathrm{RSAL}^{1}\right)\right]$.

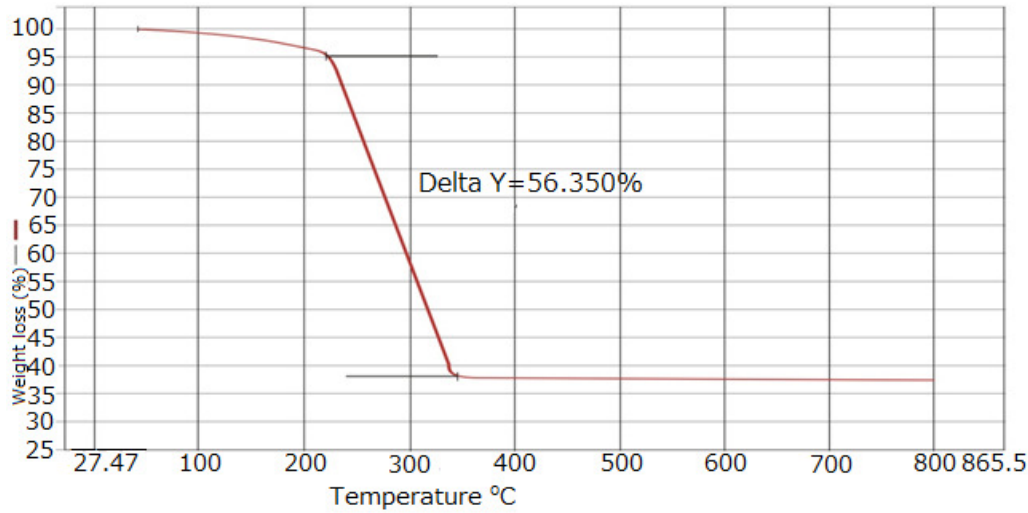

Figure 2. The TG of hydrolyzed product of $\left[\mathrm{SnO}_{2} \mathrm{AlB}\left(\mathrm{OPr}^{\mathrm{i}}\right)_{3}\left(\mathrm{RSAL}^{1}\right)\right]$. 
Table 5. Study of thermograms of hydrolyzed product of various salicylate derivatives of $\left[\mathrm{SnO}_{2} \mathrm{AlB}\left(\mathrm{OPr}^{\mathrm{i}}\right)_{4}\right]$.

\begin{tabular}{|c|c|c|c|}
\hline S. No. & Compound & Temperature range $\left({ }^{\circ} \mathrm{C}\right)$ & Weight loss (\%) \\
\hline 1. & {$\left[\mathrm{SnO}_{2} \mathrm{AlB}\left(\mathrm{OPr}^{\mathrm{i}}\right)_{3}\left(\mathrm{RSAL}^{1}\right)\right]$} & $\begin{array}{l}\text { (a) } 55-220 \\
\text { (b) } 220-345 \\
\text { (c) }>345\end{array}$ & $\begin{array}{l}\text { (a) } 5 \\
\text { (b) } 56.35 \\
\text { (c) No significant loss }\end{array}$ \\
\hline 2. & {$\left[\mathrm{SnO}_{2} \mathrm{AlB}\left(\mathrm{OPr}^{\mathrm{i}}\right)_{2}\left(\mathrm{RSAL}^{1}\right)_{2}\right]$} & $\begin{array}{l}\text { (a) } 54-222 \\
\text { (b) } 222-349 \\
\text { (c) }>349\end{array}$ & $\begin{array}{l}\text { (a) } 6 \\
\text { (b) } 63.54 \\
\text { (c) No significant loss }\end{array}$ \\
\hline 3. & {$\left[\mathrm{SnO}_{2} \mathrm{AlB}\left(\mathrm{OPr}^{\mathrm{i}}\right)_{3}\left(\mathrm{RSAL}^{2}\right)\right]$} & $\begin{array}{l}\text { (a) } 58-219 \\
\text { (b) } 219-352 \\
\text { (c) }>352\end{array}$ & $\begin{array}{l}\text { (a) } 6 \\
\text { (b) } 56.48 \\
\text { (c) No Significant loss }\end{array}$ \\
\hline 4. & {$\left[\mathrm{SnO}_{2} \mathrm{AlB}\left(\mathrm{OPr}^{\mathrm{i}}\right)_{2}\left(\mathrm{RSAL}^{2}\right)_{2}\right]$} & $\begin{array}{l}\text { (a) } 56-221 \\
\text { (b) } 221-355 \\
\text { (c) }>355\end{array}$ & $\begin{array}{l}\text { (a) } 4 \\
\text { (b) } 65.08 \\
\text { (c) No significant loss }\end{array}$ \\
\hline 5 . & {$\left[\mathrm{SnO}_{2} \mathrm{AlB}\left(\mathrm{OPr}^{\mathrm{i}}\right)_{3}\left(\mathrm{RSAL}^{3}\right)\right]$} & $\begin{array}{l}\text { (a) } 57-220 \\
\text { (b) } 220-348 \\
\text { (c) }>348\end{array}$ & $\begin{array}{l}\text { (a) } 4 \\
\text { (b) } 61.77 \\
\text { (c) No significant loss }\end{array}$ \\
\hline 6. & {$\left[\mathrm{SnO}_{2} \mathrm{AlB}\left(\mathrm{OPr}^{\mathrm{i}}\right)_{2}\left(\mathrm{RSAL}^{3}\right)_{2}\right]$} & $\begin{array}{l}\text { (a) } 59-225 \\
\text { (b) } 225-355 \\
\text { (c) }>355\end{array}$ & $\begin{array}{l}\text { (a) } 6 \\
\text { (b) } 66.40 \\
\text { (c) No significant loss }\end{array}$ \\
\hline 7. & {$\left[\mathrm{SnO}_{2} \mathrm{AlB}\left(\mathrm{OPr}^{\mathrm{i}}\right)_{3}\left(\mathrm{RSAL}^{4}\right)\right]$} & $\begin{array}{l}\text { (a) } 56-224 \\
\text { (b) } 224-350 \\
\text { (c) }>350\end{array}$ & $\begin{array}{l}\text { (a) } 5 \\
\text { (b) } 62.57 \\
\text { (c) No significant loss }\end{array}$ \\
\hline 8. & {$\left[\mathrm{SnO}_{2} \mathrm{AlB}\left(\mathrm{OPr}^{\mathrm{i}}\right)_{2}\left(\mathrm{RSAL}^{4}\right)_{2}\right]$} & $\begin{array}{l}\text { (a) } 61-230 \\
\text { (b) } 230-355 \\
\text { (c) }>355\end{array}$ & $\begin{array}{l}\text { (a) } 4 \\
\text { (b) } 71.43 \\
\text { (c) No significant loss }\end{array}$ \\
\hline
\end{tabular}

\section{CONCLUSION}

The aforesaid analytical studies suggest the substitution of terminal isopropoxy groups by salicylates but not the bridging isopropoxy groups. The proposed structures of salicylate derivatives of the type $\left[\mathrm{SnO}_{2} \mathrm{AlB}\left(\mathrm{OPr}^{\mathrm{i}}\right)_{3}(\mathrm{RSAL})\right]$ and $\left[\mathrm{SnO}_{2} \mathrm{AlB}\left(\mathrm{OPr}^{\mathrm{i}}\right)_{2}(\mathrm{RSAL})_{2}\right]$ are given as Figure 3 and Figure 4. Thermal study favors the formation of multicomponent oxides from the hydrolyzed product of salicylate derivatives.

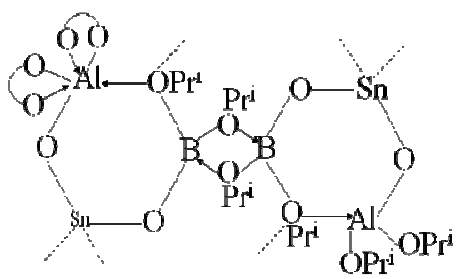

Figure 3. Plausible structure of $\left[\mathrm{SnO}_{2} \mathrm{AlB}\left(\mathrm{OPr}^{\mathrm{i}}\right)_{3}(\mathrm{RSAL})\right]$.

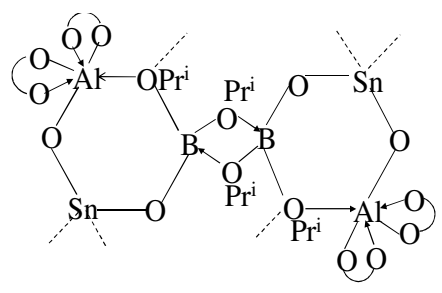

Figure 4. Plausible structure of $\left[\mathrm{SnO}_{2} \mathrm{AlB}\left(\mathrm{OPr}^{\mathrm{i}}\right)_{2}(\mathrm{RSAL})_{2}\right]$.

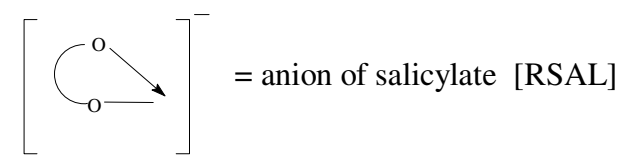

Bull. Chem. Soc. Ethiop. 2013, 27(3) 


\section{ACKNOWLEDGEMENT}

The author expresses gratitude to the Haryana Institute of Engineering \& Technology, Kaithal for the necessary facilities to accomplish this work.

\section{REFERENCES}

1. Vayssieres, L.; Hagfeldt, A.; Lindquist, S.E. Pure Appl. Chem. 2000, 72, 47.

2. Kapoor, P.N.; Bhagi, A.K.; Mulukutla, R.S.; Klabunde, K.J. Dekker Encyclopedia of Nanoscience and Nanotechnology, Marcel Dekker, Inc: NewYork; 2004.

3. Kapoor, P.N.; Sharma, H.K.; Bhagi, A.K.; Sharma, M. J. Ind. Chem. Soc. 2004, 81, 273.

4. Kapoor, P.N.; Heroux, D.; Mulukutla, R.S.; Zaikovskii, V.; Klabunde, K.J. J. Mater. Chem.2003, 13, 410.

5. Sharma, H.K.; Kumar, R. Indian J. Chem. 2008, 47A, 854.

6. Manzer, L.E.; Kourtakis, K. WO Pat. Appl. 99-US18962, 1999, 19990819.

7. Osgan, M.; Teyssie, P. J. Polym. Sci. Polym. Lett. Ed. 1967, 5, 789.

8. Carnes, C.L.; Kapoor, P.N.; Klabunde, K.J.; Bonevich, J. Chem. Mater. 2002, 14, 2922.

9. Wagner, G.W.; Procell, L.R.; O’Connor, R.J.; Munavalli, S.; Carnes, C.L.; Kapoor, P.N.; Klabunde, K.J. J. Am. Chem. Soc. 2001, 123, 1636.

10. Medine, G.M.; Zaikovskii, V.; Klabunde, K.J. J. Mater. Chem. 2004, 14, 757.

11. Klabunde, K.J.; Stark, J.V.; Koper, O.; Mohs, C.; Park, D.G.; Decker, S.; Jiang, Y.; Lagadic, I.; Zhang, D. J. Phys. Chem. 1996, 100, 12142.

12. Stark, J.V.; Park, D.G.; Lagadic, I.; Klabunde, K.J. Chem. Mater. 1996, 8, 1904.

13. Koper, O.; Lagadic, I.; Klabunde, K.J. Chem. Mater. 1997, 9, 838.

14. Klabunde, K.J. US Pat. 1999, 5990373.

15. Koper, O.; Klabunde, K.J. US Pat. 2000, 6057488.

16. Jiang, Y.; Decker, S.; Mohs, C.; Klabunde, K.J. J. Catal. 1998, 180, 24.

17. Bhagat, M.; Singh, A.; Mehrotra, R.C. Synth. React. Inorg. Metal-org. Chem. 1998, 28, 997.

18. Mehrotra, R.C.; Singh, A.; Bhagat, M.; Godhwani, J. J. Sol-gel. Sci\&Tech. 1998, 13, 45.

19. Sharma, H.K. Kapoor, P.N. Indian J. Chem. 2004, 43A, 556.

20. Sharma, M.; Bhagi, A.K.; Priti; Kansal, R.; Kumar, R.; Kapoor, P.N. Indian J. Chem. 2005, 44A, 1

21. Turova, N.Ya. Russian Chem. Rev. 2004, 73, 1041.

22. Shcheglov, P.A.; Drobot, D.V. Russian Chem. Bull. 2005, 54, 2247

23. Kumar, R. E-J. Chem. 2012, 9, 1058.

24. Kumar, R.; Sharma, H.K. J. Korean Chem. Soc. 2012, 56, 54

25. Sharma, H.K.; Kapoor, P.N. Polyhedron 1988, 7, 1389.

26. Vogel, A.I. A Text Book of Quantitative Analysis, Longman: London; 1989.

27. Perrin, D.D.; Armarego, W.L.F.; Perrin, D.R. Purification of Laboratory Chemicals, 2nd ed., Pergamon Press: New York; 1980.

28. Bradley, D.C.; Halim, F.M.A.; Wardlaw, W. J. Chem. Soc. 1950, 3450.

29. Osipov, O.A.; Verknovodova, V.I.M; Knyazhanskii, M.I. Zh. Neorg Khim. 1967, 12, 1549.

30. Mayadeo, M.S. J. Ind. Chem. Soc. 1967, 11, 502.

31. Kumar, R.; Sharma, H.K. Inorg. Chem.: An Indian J. 2008, 3, 218.

32. Nakamoto, K. Infrared and Raman Spectra of Inorganic and Coordination Compounds, John Wiley and Sons: New York; 1986.

33. Brcitmair, E.; Voelter, W. ${ }^{13}$ C NMR Spectroscopy (High Resolution Methods and Application in Organic Chemistry and Biochemistry), VCH: New York; 1990.

34. Webb, G.A. Nucl. Magn. Reson. 2009, 38, 125 
35. Wang, X.; Wang, C.; Zhao, H. Int. J. Mol. Sci. 2012, 13, 15420

36. Nguyen, T.T.; Ogwuru, N.; Eng, G. App. Org. Chem. 2000, 14, 345.

37. Hani, R.; Geanangel, R.A. Coord. Chem. Rev. 1982, 44, 229

38. Oliveira, R.A.; Silva, R.O.; Molander, G.A.; Menezes, P.H. Magn. Reson. Chem. 2009, 47, 873.

39. Mirzaee, M.; Amini, M.M.; Sadeghi, M.; Mousavi, F.Y.; Sharbatdaran, M. Ceram. Silikáty. 2005, 49,40 